\title{
ON EXTREMAL MEASURES FOR CONSERVATIVE PARTICLE SYSTEMS 尔
}

\author{
Sunder SETHURAMAN \\ Department of Mathematics, Iowa State University, 400 Carver Hall, Ames, IA 50010, USA
}

Received in 23 October 1997, revised 21 February 2000

\begin{abstract}
It is well known that the exclusion, zero-range and misanthrope particle systems possess families of invariant measures due to the mass conservation property. Although these families have been classified a great deal, a full characterization of their extreme points is not available. In this article, we consider an approach to the study of this classification. One of the results in this note is that the zero-range product invariant measures, $\prod_{i \in S} \mu_{\alpha(\cdot)}$, for an infinite countable set $S$, under mild conditions, are identified as extremal for $\alpha(\cdot) \in H_{Z R}$ where $\mu_{\alpha(i)}(k)=Z(\alpha(i))^{-1} \alpha(i)^{k} / g(1) \cdots g(k)$ with $g$ and $Z$ the rate function and normalization respectively, and $H_{Z R}$ is the set of invariant measures for the transition probability $p$. ( $) 2001$ Éditions scientifiques et médicales Elsevier SAS
\end{abstract}

Keywords: Simple exclusion process; Zero-range process; Misanthrope process; Invariant measures; Extreme points; Dirichlet form

AMS classification: Primary 60K35; secondary 60F05

RÉSUMÉ. - Il est bien connu que le procesus d'exclusion, le processus de zero-range et le processus des misanthropes possdent des familles de mesures invariantes, en raison de la propriete de conservation de la masse. Bien que ces familles aient ete beaucoup etudiees, il n'existe pas de caracterisation complete de leurs points extremaux. Dans cet article, nous considerons une approche de cette clarification. L'un de nos resultats etablit pour le processus de zero-range avec un ensemble denombrable de sites $S$ que les mesures invariantes produits $\prod_{i \in S} \mu_{\alpha(\cdot)}$ (ou $\mu_{\alpha(i)}(k)=Z(\alpha(i))^{-1} \alpha(i)^{k} / g(1) \cdots g(k), g$ etant la function de taux et $Z$ la normalisation) sont extremales pour $\alpha(\cdot) \in H_{Z R}, H_{Z R}$ designant l'ensemble des mesures invariantes pour la probabilite de transition $p$. \ 2001 Éditions scientifiques et médicales Elsevier SAS

\section{Introduction and results}

We describe an approach from the "folklore" to study ergodic properties of conservative interacting particle systems. This method, in particular, applies to the zero-range,

\footnotetext{
${ }^{17}$ Research supported in part by NSF grant NSF/DMS-9703811.

E-mail address: sethuram@iastate.edu (S. Sethuraman).
} 
misanthrope and simple exclusion processes discussed in [3,5] and [10]. Some of the main interest in such particle systems is that they possess infinite families of invariant measures. This is intuitive - since no particles die or are born, one expects collections of equilibria, each equilibrium supported on configurations of a fixed particle density.

One of the interesting questions, then, is to classify all the invariant measures. Or equivalently to characterize the extremals in the convex set of invariant measures. Besides being important in the study of the process invariance properties, these extremal measures are significant in understanding the process time-ergodic properties: When initial configurations are governed by an extreme measure, the process evolution is ergodic with respect to the time shifts [13] (see also [1] in this connection). Part of the motivation behind this note are some applications which would follow from an ergodic theorem. Determining all the extremal measures in all cases, however, seems difficult. In this article, we prove that a wide class of explicitly known invariant measures for zero-range and misanthrope systems are extremal in lieu of a complete classification. The method discussed also applies to exclusion processes, but given their rather studied characterization [10], no new extensions are presented here. As a comparison to the other systems, however, we list some of the related exclusion results.

We now introduce some notation: Let $\Sigma$ be the configuration space corresponding to an infinite countable set $S$. For exclusion dynamics, $\Sigma=\{0,1\}^{S}$, and for zero-range and misanthrope dynamics, $\Sigma=N^{S}$ where $N=\{0,1,2, \ldots\}$. Let $\eta(t)=\left\{\eta_{i}(t)\right\}_{i \in S}$ be the process configuration at time $t$ where $\eta_{i}(t)$ is the number of particles at $i \in S$ at time $t$. Let also $\{p(i, j)\}$ for $i, j \in S$ be the single particle jump probabilities. The exclusion, zero-range, and misanthrope systems are Markovian processes generated by the infinitesimal operators $L^{S E}, L^{Z R}$, and $L^{M}$ respectively acting on test functions $\phi$ :

$$
\begin{aligned}
L^{S E} \phi & =\sum \eta_{i}\left(1-\eta_{j}\right)\left(\phi\left(\eta^{i j}\right)-\phi(\eta)\right) p(i, j), \\
L^{Z R} \phi & =\sum g\left(\eta_{i}\right)\left(\phi\left(\eta^{i j}\right)-\phi(\eta)\right) p(i, j), \quad \text { and } \\
L^{M} \phi & =\sum b\left(\eta_{i}, \eta_{j}\right)\left(\phi\left(\eta^{i j}\right)-\phi(\eta)\right) p(i, j),
\end{aligned}
$$

where $\eta^{i j}=\eta-\delta_{i}+\delta_{j}$ is the new configuration which moves a particle from $i$ to $j, \delta_{k}$ is the configuration empty at all sites except for a single particle at $k$, and the functions $g(\cdot): N \rightarrow R_{+}, b(\cdot, \cdot): N \times N \rightarrow R_{+}$are known as rate functions. The construction of these systems from $L^{S E}, L^{Z R}$, and $L^{M}$, found in [10,3] and [5] respectively, require some conditions:

(SE) $\sup _{j} \sum_{i} p(i, j)<C_{p}$.

(ZR) $\sup _{k}|g(k+1)-g(k)|<C_{g}$.

(M) $b \uparrow(\downarrow)$ in the first (second) coordinate and $p(i, j)=p(j-i)$.

To avoid some complication, we assume throughout that $p(i, j)$ is irreducible; note that many statements, including Theorem 1.4, are valid under the weaker assumption $(p(i, j)+p(j, i))$ is irreducible. Also, we assume the conditons:

(ZR) $g(0)=0, \inf _{k \geqslant 1} g(k)=I>0$ and $\lim _{|i| \rightarrow \infty} p(i, j)=0$ for all $j$. 
(M) $b(0, i)=0$ for all $i$,

$$
\frac{b(i, j)}{b(j+1, i-1)}=\frac{b(i, 0) b(1, j)}{b(j+1, i-1) b(1, i-1)} \quad \text { for } i \geqslant 1, j \geqslant 0
$$

and either $p$ is symmetric or $b(i, j)-b(j, i)=b(i, 0)-b(j, 0)$ for $i, j \geqslant 0$.

When $p(i, j)$ is positive recurrent the invariant measures concentrate on configurations with finite numbers of particles, The behavior is of a different category and we do not consider this case here. Details and exact statements may be found in [10] for exclusion dynamics and [19], and [3] for the cases $g(k)=I(k \geqslant 1)$ and general $g(\cdot) \uparrow$ for zero-range dynamics; here $I(\cdot)$ is the usual indicator function.

In this note, throughout, we fix attention on the situation when $p(i, j)$ is either nullrecurrent or transient, that is the case of infinite numbers of particles. For misanthrope dynamics, this is already assumed by taking $p$ translation invariant.

We now list some of the existing results. Many rely on the double stochasticity of $p$, that is when $\sum_{j} p(i, j)=1$ for all $i$. We note when $p$ is symmetric or translation invariant, that is, $p(i, j)=p(0, j-i)$, double stochasticity is automatically satisfied. With this stochasicity condition, the exclusion, zero-range, and misanthrope processes all possess families of identically distributed product invariant measures supported on configuration hyperplanes of fixed densities $\rho$.

Denote by $B_{\rho}$ the Bernoulli product measure indexed over $S$ with coin-tossing marginal, $B_{\rho}\left\{\eta_{i}=1\right\}=1-B_{\rho}\left\{\eta_{i}=0\right\}=\rho$, all $i \in S$. Let $H_{S E}=\{0 \leqslant \rho(\cdot) \leqslant$ 1: $\left.\sum_{i} p(i, j) \rho(i)=\rho(j)\right\}$, the set of invariant measures of $p$. Define $\tilde{B}_{\rho(\cdot)}$ as the product measure over $S$ with coin-tossing marginal at site $i \in S$ with success probability $\rho(i)$ for $\rho \in H_{S E}$; then as in Theorem 8.1.24 of [10] define

$$
B_{\rho(\cdot)}=\lim _{t} \tilde{B}_{\rho} T_{t},
$$

where $T_{t}$ is the process semi-group.

For $\alpha(\cdot): S \rightarrow R_{+}$, let $Z_{\alpha(\cdot)}$ be the product measure indexed over $S$ with marginals $\mu_{\alpha(i)}$ on $N$. Here, for $\alpha>0$,

$$
\mu_{\alpha}\{k\}=\frac{1}{Z(\alpha)} \frac{\alpha^{k}}{g(1) \cdots g(k)}
$$

for $k \geqslant 1$ and $\mu_{\alpha}\{0\}=Z(\alpha)^{-1}$ where $Z(\alpha)$ is the normalization. When $\alpha=0$, set $\mu_{0}$ to be the point mass on 0 . Let also $H_{Z R}=\left\{0<\alpha(\cdot)<\liminf _{k} g(k): \sum_{i} p(i, j) \alpha(i)=\right.$ $\alpha(j)\}$.

Let $M_{\rho}$ be the product measure over $S$ with common marginal $v_{\rho}$ on $N$ satisfying

$$
\frac{v_{\rho}\{i+1\}}{v_{\rho}\{i\}}=\frac{v_{\rho}\{1\}}{v_{\rho}\{0\}} \frac{b(1, i)}{b(i+1,0)}
$$

for $\rho>0$. For $\rho=0$, let $M_{0}$ be the point mass on the configuration with no particles.

We denote by $E_{\alpha(\cdot)}$ or $E_{\rho}$ expectation with respect to these measures. 
For zero-range dynamics, we make a further assumption on $Z_{\alpha(\cdot)}$ in order to satisfy a technical condition in Theorem 1.8 of [3] and to state some of the claims below. Consider the restricted set of configurations $\Sigma^{\prime} \subset \Sigma$ defined in (2.1). We will assume that

(ZR) $Z_{\alpha(\cdot)}\left(\Sigma^{\prime}\right)=1$

We note this condition is always satisfied when $\alpha(\cdot)$ is constant or $g(k) \geqslant \delta k$, all $k \geqslant 0$; in fact, it remarked in [3], that with a different construction, with a different subset $\Sigma^{\prime}$, this condition is always true. In a sense, restricting the process to $\Sigma^{\prime}$ prevents blow-up and we refer to the discussion in Section 2 and to [3].

Let $\mathcal{I}$ denote the set of invariant measures for these processes and let $\mathcal{I}_{e}$ be the set of extremal measures in $\mathcal{I}$.

Recall the assumptions above, (SE), (ZR) and (M). We state the theorem:

THEOREM 1.1. - (1) (Liggett [10]) For simple exclusion (SE) with doubly stochastic $p,\left\{B_{\rho}:\right.$ constant $\left.\rho \in[0,1]\right\} \subset \mathcal{I}$.

(2) (Andjel [3]) For zero-range dynamics $(Z R),\left\{Z_{\alpha}: \alpha \in H_{Z R}\right\} \subset \mathcal{I}$.

(3) (Cocozza [5]) For misanthrope dynamics $(M),\left\{M_{\rho}: 0 \leqslant \rho<\infty\right\} \subset \mathcal{I}$.

There are extensions of this theorem, notably to the case when $p$ is symmetric or null-recurrent with respect to extreme points.

We have now the following (some results, when overlapping with others, are not stated in the generality found in the original papers):

THEOREM 1.2. - (1) (Liggett [10]) For simple exclusion (SE) with symmetric p, $\left\{B_{\rho(\cdot)}: \rho \in H_{S E}\right\}=\mathcal{I}_{e}$.

(2a) (Andjel [3]) For zero-range dynamics ( $Z R)$ with g increasing, and null-recurrent $p,\left\{Z_{\alpha(\cdot)}: \alpha \in H_{Z R}\right\}=\mathcal{I}_{e}$.

(2b) (Saada [15]) For zero-range dynamics $(Z R)$ with $g(k) \equiv I(k \geqslant 1)$ and transient doubly stochastic $p,\left\{Z_{\alpha}: 0 \leqslant \alpha \leqslant 1\right\} \subset \mathcal{I}_{e}$.

(2c) (Liggett [9]) For zero-range dynamics $(Z R)$ with $g(k) \equiv k$ and transient translation invariant $p,\left\{Z_{\alpha}: 0 \leqslant \alpha<\infty\right\}=\mathcal{I}_{e}$.

(3) (Andjel, Cocozza and Roussignol [4]) For misanthrope dynamics $(M)$ in $d=1$ such that $\sum i p(i)=0$ and $\sum|i| p(i)<\infty,\left\{M_{\rho}: 0 \leqslant \rho<\infty\right\}=\mathcal{I}_{e}$.

An important result which identifies a situation when a particular $B_{\rho}, Z_{\alpha}$, or $M_{\rho}$, for constant $\rho$ and $\alpha$, is extremal is the following theorem due to Saada [15]. Suppose the operators $A, A^{*}$ and $A+A^{*}$, where $A^{*}$ is the $L^{2}$ adjoint of $A$, generate conservative particle system semi-groups.

THEOREM 1.3. - Suppose $v$ is invariant for the processes generated by the operators $A$ and $A^{*}$. Assume also that $v$ is extremal for the symmetrized process operator $A+A^{*}$. Then we have that $v$ is extremal for $A$ and $A^{*}$.

An immediate corollary, noting Theorem 1.3, which fills in more pieces is the following:

COROLlary 1.1. - (1) Under (SE), for doubly stochastic $p,\left\{B_{\rho}: 0 \leqslant \rho \leqslant 1\right\} \subset \mathcal{I}_{e}$.

(2) Under $(Z R)$, for doubly stochastic $p,\{(p(i, j)+p(j, i)) / 2\}$ null-recurrent, $g$ increasing, and for constant $\alpha, Z_{\alpha} \in \mathcal{I}_{e}$. 
(3) For misanthrope processes $(M)$ in $d=1, M_{\rho} \in \mathcal{I}_{e}$ when $b(i, j)-b(j, i)=$ $b(i, 0)-b(j, 0)$ for $i, j \geqslant 0$ and $\sum|i| p(i)<\infty$.

The new results in this note focus on zero-range and misanthrope systems; as remarked earlier, nothing new is added for simple exclusion. In combination with some of the previous statments, for clarity, they are:

THEOREM 1.4. - (1) For zero-range dynamics $(Z R),\left\{Z_{\alpha(\cdot)}: \alpha(\cdot) \in H_{Z R}\right\} \subset \mathcal{I}_{e}$.

(2) For misanthrope dynamics $(M),\left\{M_{\rho}: 0 \leqslant \rho<\infty\right\} \subset \mathcal{I}_{e}$.

Our main contribution is that we do not need null-recurrence of $p$ or increasing assumptions on $g$ to determine the extremality for zero-range processes of $Z_{\alpha(\cdot)}$ for $\alpha(\cdot) \in H_{Z R}$. Also, for misanthrope systems, no assumptions beyond (M) are required to ensure extremality of $M_{\rho}$ for $\rho$ constant.

Part of what remains as an open problem is to show that the set $\left\{Z_{\alpha(\cdot)}: \alpha(\cdot) \in H_{Z R}\right\}$ exactly comprises the set of extremals $\mathcal{I}_{e}$. We have not attempted a full summary of the existing results and we refer to Chapter 8 of [10], [3] and [4] for further results.

The article is organized as follows: In Section 2, we describe the Dirichlet-form technique in the "folklore" to prove parts (1) and (2) of Theorem 1.4. In Section 3, we present a few simple applications.

\section{Dirichlet-form approach}

In this section, we discuss a technique in the "folklore" to prove extremality of invariant measures utilizing the Dirichlet form of the process. In this connection, see Sections 4.3, 4.4 of [10] with respect to Glauber dynamics, Section II.1.1.3 of [18] with respect to dynamics with Gibbs invariant measures, and the last lemma of [8] with respect to interacting Brownian particles. For clarity, we concentrate on the zero-range process and prove part (1) of Theorem 1.4, although the arguments also apply to simple exclusion and misanthrope systems. Perhaps of specific interest is that the approach here applies to inhomogeneous measures $Z_{\alpha(\cdot)}$.

We recall now some of the details of the zero-range construction in [3] and extend the process to $L^{2}$. Different from simple exclusion systems, the process semi-group $T_{t}$ is not constructed from Hille-Yosida considerations. Rather, $T_{t} f(\eta)=E_{\eta}[f(\eta(t))]$ is shown to exist on a class of functions $\mathcal{L}$ defined on a subset $\Sigma^{\prime} \subset \Sigma$ of the configuration space. Following [3], for fixed $g$ and $p$, let $\beta(i)=\sum_{n=0}^{\infty} 2^{-n} p^{n}(i, 0)$. We can take

$$
\begin{gathered}
\Sigma^{\prime}=\left\{\eta:\|\eta\|=\sum_{i \in S}\left|\eta_{i}\right| \beta(i)<\infty\right\}, \quad \text { and } \\
\mathcal{L}=\left\{f:|f(\eta)-f(\xi)| \leqslant c\|\eta-\xi\| \text { for all } \eta, \xi \in \Sigma^{\prime}, \text { some } c\right\} ;
\end{gathered}
$$

for $f \in \mathcal{L}$, let $L(f)$ be the smallest such constant $c$. Let also $\mathcal{L}^{\prime} \subset \mathcal{L}$ be the bounded functions in $\mathcal{L}$. It is proved in Lemma 2.2 of [3] that $T_{t}: \mathcal{L} \rightarrow \mathcal{L}$, and in fact $T_{t}: \mathcal{L}^{\prime} \rightarrow \mathcal{L}^{\prime}$. Formally, a measure $v$ is invariant for the process if for all $f \in \mathcal{L}^{\prime}$,

$$
\int T_{t} f d v=\int f d v
$$


The restriction of configurations to $\Sigma^{\prime}$ makes sense in that explosion should be ruled out. In fact, by assumption (ZR), the invariant measures $Z_{\alpha(\cdot)}$ for $\alpha(\cdot) \in H_{Z R}$ are all supported on $\Sigma^{\prime}$, and it can be shown that the process never leaves $\Sigma^{\prime}$.

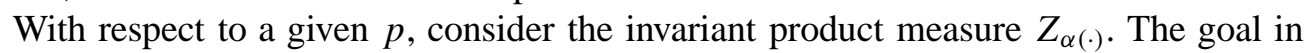
the next series of lemmas is to extend the process to $L^{2}\left(Z_{\alpha(\cdot)}\right)$.

Define now the "triple norm" function, \|\|$\cdot\|\mid\|: \Sigma^{\prime} \rightarrow R$ by $\left\||\eta \||=\sum g\left(\eta_{i}\right) \beta(i)\right.$. Fix also $S_{n}$ be an increasing sequence of finite subsets of $S$ such that $S=\bigcup_{n \geqslant 1} S_{n}$. Note that $\sum_{j} p(i, j) \beta(j) \leqslant 2 \beta(i)$ and that, for $f \in \mathcal{L}$, we have

$$
\begin{aligned}
\left|g\left(\eta_{i}\right)\left(f\left(\eta^{i j}\right)-f(\eta)\right) p(i, j)\right| & \leqslant L(f) g\left(\eta_{i}\right) p(i, j)(\beta(i)+\beta(j) \\
& \leqslant L(f) C_{g}\left|\eta_{i}\right| p(i, j)(\beta(i)+\beta(j)),
\end{aligned}
$$

so that for $\eta \in \Sigma^{\prime}, L^{Z R} f(\eta)$ is well defined and

$$
\left|L^{Z R} f(\eta)\right| \leqslant 3 L(f)\left|\|\eta \mid\| \leqslant 3 L(f) C_{g}\|\eta\| .\right.
$$

Take note also, from Lemma 3.2 of [3], that

$$
E_{\alpha(\cdot)}[\||| \eta|| \mid]=\sum_{i} \alpha(i) \beta(i)<\infty .
$$

In fact,

LEMMA 2.1. - $\|\mid \eta\| \| \in L^{2}\left(Z_{\alpha(\cdot)}\right)$.

Proof. - As the $\left\{g\left(\eta_{i}\right): i \in S\right\}$ are non-negative and independent, write

$$
\begin{aligned}
E_{\alpha(\cdot)}\left[(\|\| \eta \|||)^{2}\right] & =\sum_{i, j} E_{\alpha(\cdot)}\left[g\left(\eta_{i}\right) g\left(\eta_{j}\right)\right] \beta(i) \beta(j) \\
& =\sum_{i \neq j} \alpha(i) \beta(i) \alpha(j) \beta(j)+\sum_{i} E_{\alpha(\cdot)}\left[g\left(\eta_{i}\right)^{2}\right] \beta(i)^{2} .
\end{aligned}
$$

Observe that $E_{\alpha(\cdot)}\left[g\left(\eta_{i}\right)^{2}\right]=\alpha(i) E_{\alpha(\cdot)}\left[g\left(\eta_{i}+1\right)\right]$. So that the last sum above, through the Lipshitz bound (ZR) on $g$ after adding and subtracting $g\left(\eta_{i}\right)$, is bounded above by

$$
\sum_{i} \alpha(i) E_{\alpha(\cdot)}\left[g\left(\eta_{i}+1\right)\right] \beta(i)^{2} \leqslant \sum_{i} C_{g} \alpha(i) \beta(i)^{2}+\sum_{i} \alpha(i)^{2} \beta(i)^{2} .
$$

Noting $0 \leqslant \beta(i) \leqslant 1$ and $(2.3)$ gives

$$
E_{\alpha(\cdot)}\left[(|||\eta|||)^{2}\right] \leqslant \max \left(C_{g}, 1\right) E_{\alpha(\cdot)}[|||\eta|||]\left(1+E_{\alpha(\cdot)}[|||\eta|||]\right)<\infty
$$

to finish the lemma.

The following discussion borrows from pp. 205-208, Chapter 4 in [10]; certain proofs are included here for completeness.

LEMMA 2.2. $-T_{t}$ on $\mathcal{L}^{\prime}$ extends by continuity to a Markov semi-group $T_{t}^{\alpha}$ on $L^{2}\left(Z_{\alpha(\cdot)}\right)$. 
Proof. - For $f \in \mathcal{L}^{\prime}$, let $T_{t} f(\eta)=E_{\eta}[f(\eta(t))]$ be the conditional expectation of $f$, so that by Schwarz inequality,

$$
\left[T_{t} f(\eta)\right]^{2} \leqslant T_{t} f^{2}(\eta)
$$

Then

$$
\begin{aligned}
\left\|T_{t} f\right\|_{L^{2}(\alpha)}^{2} & =\int\left[T_{t} f\right]^{2} d Z_{\alpha(\cdot)} \\
& \leqslant \int T_{t} f^{2} d Z_{\alpha(\cdot)} \\
& =\int f^{2} d Z_{\alpha(\cdot)}=\|f\|_{L^{2}(\alpha(\cdot))} .
\end{aligned}
$$

As simple functions are contained in $\mathcal{L}^{\prime}, \mathcal{L}^{\prime}$ is dense in $L^{2}(\alpha(\cdot))$. Therefore $T_{t}$ extends to a Markov semi-group $T_{t}^{\alpha}$ on $L^{2}(\alpha)$.

Let now $L_{\alpha}^{Z R}$ be the Markov generator associated to $T_{t}^{\alpha}$ acting on the following domain:

$$
\operatorname{Dom}(\alpha)=\left\{f: \lim _{t \rightarrow 0} \frac{T_{t}^{\alpha} f-f}{t} \text { exists in } L^{2}(\alpha(\cdot))\right\} .
$$

Lemma 2.3. - We have $\mathcal{L}^{\prime} \subset \operatorname{Dom}(\alpha)$ and therefore $L_{\alpha}^{Z R}$ on $\operatorname{Dom}(\alpha)$ extends $L^{Z R}$ on $\mathcal{L}^{\prime}$. In fact, $L_{\alpha}^{Z R}$ is the closure of $L^{Z R}$ on $\mathcal{L}^{\prime}$.

Proof. - Let $f \in \mathcal{L}^{\prime}$ and $\eta \in \Sigma^{\prime}$. It is proved just after Lemma 2.6 of [3] that

$$
L\left(T_{t} f\right) \leqslant L(f) \exp \left(3 C_{g} t\right)
$$

Also, the pointwise convergence is proved in Lemma 2.7(f) of [3]:

$$
\lim _{t \rightarrow 0} \frac{T_{t} f(\eta)-f(\eta)}{t}=L^{Z R} f(\eta) .
$$

In addition, part (b) Lemma 2.7 of [3] gives that

$$
\frac{T_{t} f(\eta)-f(\eta)}{t}=\frac{1}{t} \int_{0}^{t} L^{Z R} T_{s} f(\eta) d s .
$$

This last statement, combined with (2.2), (2.4), and Lemma 2.1 yields, for $0<t \leqslant 1$, that

$$
t^{-1}\left|T_{t} f-f\right|(\eta) \leqslant C(f, g)\left|\left\|\eta|\|| \in L^{2}\left(Z_{\alpha(\cdot)}\right) .\right.\right.
$$

Also, by (2.2) and Lemma 2.1, $\left|L^{Z R} f(\eta)\right| \leqslant C_{f}\||\eta|\| \in L^{2}\left(Z_{\alpha(\cdot)}\right)$. Therefore, as $T_{t}^{\alpha}$ extends $T_{t}$ on $\mathcal{L}^{\prime}$, we have by dominated convergence for $f \in \mathcal{L}^{\prime}$ that

$$
\frac{1}{t}\left(T_{t}^{\alpha} f-f\right)(\eta) \rightarrow L^{Z R} f(\eta)
$$

converges in $L^{2}\left(Z_{\alpha(\cdot)}\right)$ as $t \downarrow 0$. Hence, $L_{\alpha}^{Z R}$ extends $L^{Z R}$ on $\mathcal{L}^{\prime}$ and $\mathcal{L}^{\prime} \subset \operatorname{Dom}(\alpha)$. 
Now as $T_{t}: \mathcal{L}^{\prime} \rightarrow \mathcal{L}^{\prime}$ and $\mathcal{L}^{\prime} \subset \operatorname{Dom}(\alpha)$ is dense in $L^{2}\left(Z_{\alpha(\cdot)}\right)$, we conclude by Lemma 1.3.3 of [6] that $\mathcal{L}^{\prime}$ is a core for $L_{\alpha}^{Z R}$. This finishes the proof.

We will need a certain representation of the Dirichlet form of the process. Define the Dirichlet form $D_{\alpha(\cdot)}(f)=-E_{\alpha(\cdot)}\left[f\left(L_{\alpha}^{Z R} f\right)\right]$ and $s(i, j)=\left(p(i, j)+\frac{\alpha(j)}{\alpha(i)} p(j, i)\right) / 4$.

LEMmA 2.4. - Let $f \in \operatorname{Dom}(\alpha)$. Then,

$$
D_{\alpha(\cdot)}(f)=\sum E_{\alpha(\cdot)}\left[g\left(\eta_{i}\right)\left(f\left(\eta^{i j}\right)-f(\eta)\right)^{2}\right] s(i, j)
$$

where the series converges.

Proof. - We first prove the equality for $f \in \mathcal{L}^{\prime}$ : By (2.2) and (2.3), we have that

$$
E_{\alpha(\cdot)}\left[\sum_{i, j}\left|f(\eta) g\left(\eta_{i}\right)\left(f\left(\eta^{i j}\right)-f(\eta)\right) p(i, j)\right|\right] \leqslant E_{\alpha(\cdot)}\left[C_{f}|||\eta|||\right]<\infty
$$

so that by Fubini's theorem,

$$
D_{\alpha(\cdot)}(f)=-\sum_{i, j} E_{\alpha(\cdot)}\left[f(\eta) g\left(\eta_{i}\right)\left(f\left(\eta^{i j}\right)-f(\eta)\right) p(i, j)\right] .
$$

First note that the sum on the right side is absolutely summable therefore permitting rearrangement. Let now $\nabla_{x y} h(\eta)=h\left(\eta^{x y}\right)-h(\eta)$, and let also $a(x, y)=p(x, y)-$ $\frac{\alpha(y)}{\alpha(x)} p(y, x)$. Observe, with the Radon-Nikodym derivative corresponding to change of variables $\eta$ to $\xi=\eta^{y x}$,

$$
\frac{d Z_{\alpha(\cdot)}\{\xi\}}{d Z_{\alpha(\cdot)}\{\eta\}}=\frac{\mu_{\alpha(y)}\left(\eta_{y}-1\right) \mu_{\alpha(x)}\left(\eta_{x}+1\right)}{\mu_{\alpha(y)}\left(\eta_{y}\right) \mu_{\alpha(x)}\left(\eta_{x}\right)}=\frac{g\left(\eta_{y}\right)}{g\left(\eta_{x}+1\right)} \frac{\alpha(x)}{\alpha(y)}
$$

and therefore the relation

$$
E_{\alpha(\cdot)}\left[f(\eta) g\left(\eta_{x}\right)\left(\nabla_{x y} f(\eta)\right) p(x, y)\right]=-E_{\alpha(\cdot)}\left[f\left(\eta^{y x}\right) g\left(\eta_{y}\right)\left(\nabla_{y x} f(\eta)\right) \frac{\alpha(x)}{\alpha(y)} p(x, y)\right] .
$$

Now, from the sum (2.5), add together the $i j$ and $j i$ pairs and calculate:

$$
\begin{aligned}
E_{\alpha(\cdot)} & {\left[f(\eta) g\left(\eta_{i}\right)\left(\nabla_{i j} f(\eta)\right) p(i, j)\right]+E_{\alpha(\cdot)}\left[f(\eta) g\left(\eta_{j}\right)\left(\nabla_{j i} f(\eta)\right) p(j, i)\right] } \\
= & -E_{\alpha(\cdot)}\left[f\left(\eta^{j i}\right) g\left(\eta_{j}\right)\left(\nabla_{j i} f(\eta)\right) \frac{\alpha(i)}{\alpha(j)} p(i, j)\right]+E_{\alpha(\cdot)}\left[f(\eta) g\left(\eta_{j}\right)\left(\nabla_{j i} f(\eta)\right) p(j, i)\right] \\
= & -E_{\alpha(\cdot)}\left[g\left(\eta_{j}\right)\left(\nabla_{j i} f(\eta)\right)^{2} \frac{\alpha(i)}{\alpha(j)} p(i, j)\right] \\
& +E_{\alpha(\cdot)}\left[f(\eta) g\left(\eta_{j}\right)\left(\nabla_{j i} f(\eta)\right)\left(p(j, i)-\frac{\alpha(i)}{\alpha(j)} p(i, j)\right)\right] .
\end{aligned}
$$

Note that the calculation above is invariant under interchange of $i$ and $j$ so that the pair-sum also equals

$$
-E_{\alpha(\cdot)}\left[g\left(\eta_{i}\right)\left(\nabla_{i j} f(\eta)\right)^{2} \frac{\alpha(j)}{\alpha(i)} p(j, i)\right]+E_{\alpha(\cdot)}\left[f(\eta) g\left(\eta_{i}\right)\left(\nabla_{i j} f(\eta)\right) a(i, j)\right] .
$$


Adding half of this line and half of the previous last line gives that the pair sum becomes

$$
\begin{aligned}
& -(1 / 2)\left\{E_{\alpha(\cdot)}\left[g\left(\eta_{j}\right)\left(\nabla_{j i} f(\eta)\right)^{2} \frac{\alpha(i)}{\alpha(j)} p(i, j)\right]+E_{\alpha(\cdot)}\left[g\left(\eta_{i}\right)\left(\nabla_{i j} f(\eta)\right)^{2} \frac{\alpha(j)}{\alpha(i)} p(j, i)\right]\right\} \\
& +(1 / 2)\left\{E_{\alpha(\cdot)}\left[f(\eta) g\left(\eta_{j}\right)\left(\nabla_{j i} f(\eta)\right) a(j, i)\right]+E_{\alpha(\cdot)}\left[f(\eta) g\left(\eta_{i}\right)\left(\nabla_{i j} f(\eta)\right) a(i, j)\right]\right\} .
\end{aligned}
$$

Note that the separate summations of all the expectations are absolutely summable from the same method as in the beginning of the proof.

Now rewrite the first line as half of the present form and half of the form changed by transforming $\eta$ to $\eta^{i j}$ and $\eta^{j i}$ respectively in the two expectations to obtain

$$
-E_{\alpha(\cdot)}\left[g\left(\eta_{i}\right)\left(\nabla_{i j} f(\eta)\right)^{2} s(i, j)\right]-E_{\alpha(\cdot)}\left[g\left(\eta_{j}\right)\left(\nabla_{j i} f(\eta)\right)^{2} s(j, i)\right] .
$$

Observe that the sum of these $i j$ pairs is the desired result.

What remains is to show that sum of the terms in the second line vanish. To this end, write the term in brackets, by change of variables $\eta$ to $\eta^{i j}$ and the formula $\frac{\alpha(j)}{\alpha(i)} a(j, i)=-a(i, j)$ for the first expectation, and then change $\eta$ to $\eta^{j i}$ to get the last line below:

$$
\begin{aligned}
& E_{\alpha(\cdot)}\left[\left(f\left(\eta^{i j}\right)+f(\eta)\right) g\left(\eta_{i}\right)\left(\nabla_{i j} f(\eta)\right) a(i, j)\right] \\
& \quad=E_{\alpha(\cdot)}\left[\left(f^{2}\left(\eta^{i j}\right)-f^{2}(\eta)\right) g\left(\eta_{i}\right) a(i, j)\right] \\
& \quad=E_{\alpha(\cdot)}\left[\left(f^{2}\left(\eta^{i j}\right)-f^{2}(\eta)\right) g\left(\eta_{i}\right) p(i, j)\right]+E_{\alpha(\cdot)}\left[\left(f^{2}\left(\eta^{j i}\right)-f^{2}(\eta)\right) g\left(\eta_{j}\right) p(j, i)\right] .
\end{aligned}
$$

Noting that $f^{2} \in \mathcal{L}^{\prime}$, observe that the $i j$ sum of the last expression is $E_{\alpha(\cdot)}\left[L^{Z R} f^{2}\right]=0$ from Lemma 2.9 [3].

All this proves the lemma for $f \in \mathcal{L}^{\prime}$.

We now extend the representation to $\operatorname{Dom}(\alpha)$ : Let

$$
R(f)=\sum E_{\alpha(\cdot)}\left[g\left(\eta_{i}\right)\left(f\left(\eta^{i j}\right)-f(\eta)\right)^{2}\right] s(i, j)
$$

for $f \in \operatorname{Dom}(\alpha)$ such that the series converges. For $f \in \operatorname{Dom}(\alpha)$, take $f_{n} \in \mathcal{L}^{\prime}$ so that $f_{n} \rightarrow f$ and $L_{\alpha}^{Z R} f_{n} \rightarrow L_{\alpha}^{Z R} f$ in $L^{2}(\alpha(\cdot))$. Then

$$
\begin{gathered}
\lim _{n \rightarrow \infty} D_{\alpha(\cdot)}\left(f_{n}\right)=D_{\alpha(\cdot)}(f), \quad \text { and } \\
\liminf _{n \rightarrow \infty} R\left(f_{n}\right) \geqslant R(f)
\end{gathered}
$$

by Fatou's lemma. Therefore, $R(f) \leqslant D_{\alpha(\cdot)}(f)$ and in particular, $R(f)<\infty$ for $f \in$ $\operatorname{Dom}(\alpha)$. However also,

$$
0 \leqslant D_{\alpha(\cdot)}\left(f-f_{n}\right) \leqslant\left\|f-f_{n}\right\|_{L^{2}} \cdot\left\|L_{\alpha}^{Z R} f-L_{\alpha}^{Z R} f_{n}\right\|_{L^{2}}
$$

which vanishes as $n \rightarrow \infty$. Hence, $\lim _{n \rightarrow \infty} R\left(f-f_{n}\right)=0$, and so $\lim _{n \rightarrow \infty} R\left(f_{n}\right)=$ $R(f)$, to finish the proof.

We will need the following two propositions for the proof of the main theorem: 
Consider a space $\Sigma$ with Borel sets $\mathcal{B}$. Let $Q$ be an invariant probability measure on $\Sigma$ for the Markov process $\{\eta(t), t \geqslant 0\}$. Let $P^{Q}$ be the probability on the path space with initial distribution $Q$ and $T_{t}$ be the process semi-group. As $T_{t}$ is a contraction on $L^{2}(Q)$, we have for $f \in L^{2}(Q)$ that

$$
\frac{1}{t} \int_{0}^{t}\left(T_{s} f\right) d s \rightarrow \hat{f}
$$

converges in $L^{2}(Q)$ to $\hat{f} \in L^{2}(Q)$ as $t \rightarrow \infty$. The following result, found in Section 2 of [13] (see also IV.2 [14]), provides equivalences between ergodicity of $P^{Q}$ and extremality of $Q$ in the set of invariant probability measures. For the convenience of the reader, we repeat the proof at the end of the section.

PROPOSITION 2.1. - All are equivalent:

(a) For sets $A \in \mathcal{B}, T_{t} I(A)=I(A) Q$-a.s. $\Rightarrow Q(A)=0$ or 1 .

(b) $P^{Q}$ is ergodic: For each $f \in L^{2}(Q), \hat{f}=E_{Q}[f]$, a.s. $Q$.

(c) $Q$ is extremal: If for some $0<\varepsilon<1$ and invariant probability measures $Q_{1}$ and $Q_{2}$, we have $Q=\varepsilon Q_{1}+(1-\varepsilon) Q_{2}$, then $Q=Q_{1}=Q_{2}$.

Note that part (b) may replaced by more usual definition of shift-ergodicity (see Corollary 5 in IV.2 [14]): $\Lambda$ shift-invariant $\Rightarrow P^{Q}(\Lambda)=0$ or 1 .

The following standard local limit theorem for independent (non-identically) distributed lattice valued random variables is taken from Theorem 7.4, p. 195 of [12]. The statement here differs slightly in that we have replaced the "log $N$ " term in [12] by " $\log c_{N}$ "; but the proof is the same and we refer the reader to [12].

Proposition 2.2. - Let $\left\{X_{i}\right\}$ be a sequence of independent r.v.'s taking integer values and let $S_{N}=\sum_{i=1}^{N} \eta_{i}, p_{i j}=P\left(X_{i}=j\right)$, and $\rho^{N}=\sum_{i=1}^{N} E\left(X_{i}\right)$. Suppose $p_{i 0} \geqslant$ $p_{i j}$ for all $i$ and $j$. Suppose furthermore that

$$
\begin{gathered}
c^{N}=\sum_{i}^{N} E\left(X_{i}-E X_{i}\right)^{2} \uparrow \infty, \quad \gamma^{N}=\sum_{1}^{N} E\left|X_{i}-E X_{i}\right|^{3}=\mathrm{O}\left(c^{N}\right), \\
\text { g.c.d. }\left\{m: \frac{1}{\log c^{N}} \sum_{j=1}^{N} p_{j 0} p_{j m} \rightarrow \infty\right\}=1 .
\end{gathered}
$$

Then

$$
\sup _{K}\left|\sqrt{c^{N}} P\left(S_{N}=K\right)-\frac{1}{\sqrt{2 \pi}} \exp \left\{-\frac{\left(K-\rho^{N}\right)^{2}}{2 c^{N}}\right\}\right|=\mathrm{O}\left(\frac{1}{\sqrt{c^{N}}}\right) .
$$

Proof of (1), Theorem 1.4. - From Proposition 2.1, it is enough to show every bounded harmonic function $f$ is constant $Z_{\alpha(\cdot)}$-almost surely. For bounded harmonic $f$ we see that $L_{\alpha}^{Z R} f=0$. It follows from the definition of the Dirichlet form $D_{\alpha(\cdot)}$ that $D_{\alpha(\cdot)}(f)=$ 0 . This immediately gives through Lemma 2.4 and the the positivity $g(k)>0$ for $k \geqslant 1$ that $Z_{\alpha(\cdot)}$ almost surely

$$
f\left(\eta^{i j}\right)=f(\eta) \quad \forall i, j \text { such that } s(i, j)>0 \text { when } \eta_{i} \geqslant 1 .
$$


A consequence of this property is that, if $\eta_{i j}$ denotes the configuration which exchanges values of $\eta_{i}$ and $\eta_{j}$, then, due to the irreducibility of $s(i, j)$ (as $p$ irreducible and $\alpha(\cdot)>0)$, that

$$
f\left(\eta_{i j}\right)=f(\eta) \text { for all } i, j \text { a.s. }
$$

The property (2.6) allows us, in the following claim, to conclude that $f(\eta)$ is measurable with respect to the tail $\sigma$-field of the coordinates $\left\{\eta_{i}: i \in S\right\}$. By Kolmogorov's $0-1$ law, this $\sigma$-field is trivial, hence $f$ must be constant almost surely. This finishes the proof.

Note that when $\alpha(\cdot) \equiv \alpha$ is constant, that is when $Z_{\alpha}$ is i.i.d product measure, the argument is simpler once we note that, by (2.7), $f$ is finite-permutation invariant and therefore constant $Z_{\alpha}$-a.s. by Hewitt-Savage's $0-1$ law. This easier argument, in particular, would apply to the misanthrope measures.

Also, note that the collection of sets $A$ which stay invariant by the transformation $\eta \rightarrow \eta_{i j}$ is a $\sigma$-field $\mathcal{A}$. Clearly, $\mathcal{T} \subset \mathcal{A} \subset \mathcal{H} \subset \mathcal{E}$ where $\mathcal{T}$ is the tail $\sigma$-field of the $\eta_{i}$ 's, $\mathcal{H}$ is the tail $\sigma$-field of the $H_{n}$ 's where $H_{n}=\sum_{i \in S_{n}} \eta_{i}$, and $\mathcal{E}$ is the exchangeable $\sigma$-field of the $\eta_{i}$ 's. Attempts to invoke triviality conditions for $\mathcal{H}$ and $\mathcal{E}$, discussed in [11] and [2] (see also [7] for 0-1 law statements in the context of Gibbs measures), failed. However, as shown in the claim below, for the measures $Z_{\alpha(\cdot),} \mathcal{A}=\mathcal{T}$.

Claim 2.1.-Let $f$ be bounded function satisfying property (2.6). Then $f$ is measurable with respect to the tail $\sigma$-field of $\left\{\eta_{i}: i \in S\right\}$.

Proof. - Let $\mathcal{F}_{k}=\sigma\left\{\eta_{i}, i \in S_{k}\right\}$ and define $\delta_{i}$ as the single particle configuration at $i$ and $\eta^{i}=\eta-\delta_{i}$ as the configuration with one less at $i$. Also define, for $n_{0}$ large enough, the local bounded function $h_{\varepsilon}=E_{\alpha(\cdot)}\left[f \mid \mathcal{F}_{n_{0}}\right]$ so that $\left\|f-h_{\varepsilon}\right\|_{L^{2}(\alpha(\cdot))}<\varepsilon$ for $\varepsilon>0$. Correspondingly, denote $R(\eta)=f(\eta)-h_{\varepsilon}(\eta)$ as the error difference function.

Suppose now that when $\eta_{0} \geqslant 1$,

$$
f(\eta)=f\left(\eta^{0}\right) \quad \text { a.s. } Z_{\alpha}(\cdot) ;
$$

that is, we may take away a particle at the origin without changing the function. For $f$, with this feature, the claim follows: Let $\bar{\eta}^{n}$ be the configuration which equals $\eta$ everywhere except in the set $S_{n}$ where the values are set to 0 . Given (2.8), we see that $f(\eta)=f\left(\bar{\eta}^{n}\right)$ a.s. by simply moving all particles in $S_{n}$ to the origin and then removing them one by one. This gives that $f$ is measurable with respect to the tail $\sigma$-field.

We now show that indeed $f$ satisfies (2.8). As will be seen, the fact $\sum_{i} \alpha(i)=\infty$, as $p$ is null-recurrent or transient, is essential.

We consider two cases: Let $U=\limsup _{|i| \rightarrow \infty} \alpha(i)$. In case 1 , we assume $U>0$ and in case 2, we take $U=0$. Just as a remark, we note that $U>0$ for $p$ such that $\sum_{i} p(i, j) \leqslant 1$ for all $j$ by the (maximum) principle that $\alpha(i)$ does not take on its maximum in $S$.

Case 1. Assume that $U>0$. Let $x_{h} \in S$ be a site which is outside the support of $h_{\varepsilon}(\eta)$. Now observe that when $\eta_{0} \geqslant 1,(2.6)$ gives $f\left(\eta^{0, x_{h}}\right)=f(\eta)$ a.s. and $h_{\varepsilon}\left(\eta^{0, x_{h}}\right)=h_{\varepsilon}\left(\eta^{0}\right)$.

To show (2.8), we try to control the $L^{2}$ difference: 


$$
\begin{aligned}
E_{\alpha(\cdot)}\left[\left(f(\eta)-f\left(\eta^{0}\right)\right)^{2}, \eta_{0} \geqslant 1\right] & =E_{\alpha(\cdot)}\left[\left(f\left(\eta^{0 x_{h}}\right)-f\left(\eta^{0}\right)\right)^{2}, \eta_{0} \geqslant 1\right] \\
& =E_{\alpha(\cdot)}\left[\left(h\left(\eta^{0 x_{h}}\right)+R\left(\eta^{0 x_{h}}\right)-h\left(\eta^{0}\right)-R\left(\eta^{0}\right)\right)^{2}, \eta_{0} \geqslant 1\right] \\
& \leqslant 2 E_{\alpha(\cdot)}\left[R^{2}\left(\eta^{0 x_{h}}\right), \eta_{0} \geqslant 1\right]+2 E_{\alpha(\cdot)}\left[R^{2}\left(\eta^{0}\right), \eta_{0} \geqslant 1\right] .
\end{aligned}
$$

Evidently then, controlling the terms $E_{\alpha(\cdot)}\left[R^{2}\left(\eta^{0 x_{h}}\right), \eta_{0} \geqslant 1\right]$ and $E_{\alpha(\cdot)}\left[R^{2}\left(\eta^{0}\right), \eta_{0} \geqslant 1\right]$, say in terms of $\|R\|_{L^{2}}<\varepsilon$, would be enough to finish.

To this end, recall that $\inf _{k \geqslant 1} g(k)=I>0$ and compute the following:

$$
\begin{aligned}
E_{\alpha(\cdot)}\left[R^{2}\left(\eta^{0, x_{h}}\right), \eta_{0} \geqslant 1\right] & =E_{\alpha(\cdot)}\left[\frac{\mu_{\alpha(0)}\left(\eta_{0}+1\right)}{\mu_{\alpha(0)}\left(\eta_{0}\right)} \frac{\mu_{\alpha\left(x_{h}\right)}\left(\eta_{x_{h}}-1\right)}{\mu_{\alpha\left(x_{h}\right)}\left(\eta_{x_{h}}\right)} R^{2}(\eta), \eta_{x_{h}} \geqslant 1\right] \\
& =E_{\alpha(\cdot)}\left[\frac{\alpha(0)}{g\left(\eta_{0}+1\right)} \frac{g\left(\eta_{x_{h}}\right)}{\alpha\left(x_{h}\right)} R^{2}(\eta)\right] \\
& \leqslant 2 C_{f} \frac{\alpha(0)}{I} E_{\alpha(\cdot)}\left[\frac{g\left(\eta_{x_{h}}\right)}{\alpha\left(x_{h}\right)}|R|(\eta)\right] \\
& \leqslant \frac{C(f, \alpha, g)}{\alpha\left(x_{h}\right)} \sqrt{E_{\alpha(\cdot)}\left[g^{2}\right] E_{\alpha(\cdot)}\left[R^{2}\right]} \\
& =\frac{C(f, \alpha, g)}{\alpha\left(x_{h}\right)} \sqrt{\alpha\left(x_{h}\right) E_{\alpha(\cdot)}\left[g\left(\eta_{x_{h}}+1\right)\right] E_{\alpha(\cdot)}\left[R^{2}\right]} \\
& \leqslant \frac{C(f, \alpha, g)}{\alpha\left(x_{h}\right)} \sqrt{\alpha\left(x_{h}\right)\left(C+\alpha\left(x_{h}\right)\right)}\|R\|_{L^{2} .}
\end{aligned}
$$

Above, we use in the third step the bound $\|R\|_{L^{\infty}}<C_{f}$, in the fifth step the identity $E_{\alpha(\cdot)}\left[g\left(\eta_{i}\right) f(\eta)\right]=\alpha(i) E_{\alpha(\cdot)}\left[f\left(\eta+\delta_{i}\right)\right]$ and in the sixth step the zero-range Lipschitz bound on $g(\cdot)$. Note that as $\lim \sup \alpha(i)=U>0$, we may find $x_{h}$ (no matter how large the support of $h_{\varepsilon}$ is) so that $\alpha\left(x_{h}\right)>U / 2$. Hence, the coefficient of $\|R\|_{L^{2}}$ in (2.9) may be bounded above uniformly in $x_{h}$ and we have for some constant $C$, $E_{\alpha(\cdot)}\left[R^{2}\left(\eta^{0, x_{h}}\right), \eta_{0} \geqslant 1\right]<C(f, \alpha, g)\|R\|_{L^{2}}<C(f, \alpha, g) \varepsilon$.

Similarly and more easily, $E_{\alpha(\cdot)}\left[R^{2}\left(\eta^{0}\right), \eta_{0} \geqslant 1\right] \leqslant(\alpha(0) / I) E_{\alpha(\cdot)}\left[R^{2}(\eta)\right]<C(\alpha, g) \varepsilon^{2}$.

As $\varepsilon$ is arbitrary, this finishes the proof.

Case 2. Assume $U=0$. The difficulty with the previous argument is that now we cannot control the coefficient in (2.9). So, instead of placing the particle at the site $x_{h}$ we will put it in a far region whose occupation distribution is more tractable. Recall that $\sum_{i} \alpha(i)=\infty$.

Let $h_{\varepsilon}, n_{0}$ and $R$ be as before. For $l>n>n_{0}$, define the annulus $A_{n, l} \subset S$ as the region between the sets $S_{n}$ and $S_{l}$ and denote $T_{n, l}=\sum_{i \in A_{n, l}} \eta_{i}$. Observe that the functions $f$ and $R$, as a consequence of the invariance property (2.6), depends on the variables in $A_{n, l}$ only through the sum of occupation numbers, $T_{n, l}$. In fact, for a fixed $x_{n, l} \in A_{n, l}$, let $\tau_{n, l}(\eta)$ denote the configuration where all particles in $A_{n, l}$ have been moved to $x_{n, l}$.

Beginning as in case $1, E_{\alpha(\cdot)}\left[\left(f(\eta)-f\left(\eta^{0}\right)\right)^{2}, \eta_{0} \geqslant 1\right]$ equals

$$
\begin{aligned}
& E_{\alpha(\cdot)}\left[\left(f\left(\tau_{n, l}(\eta)\right)-f\left(\eta^{0}\right)\right)^{2}, \eta_{0} \geqslant 1\right] \\
& \quad=E_{\alpha(\cdot)}\left[\left(f\left(\left(\tau_{n, l}(\eta)\right)^{0, x_{n, l}}\right)-f\left(\eta^{0}\right)\right)^{2}, \eta_{0} \geqslant 1\right] \\
& \quad \leqslant 2 E_{\alpha(\cdot)}\left[R^{2}\left(\left(\tau_{n, l}(\eta)\right)^{0, x_{n, l}}\right), \eta_{0} \geqslant 1\right]+2 E_{\alpha(\cdot)}\left[R^{2}\left(\eta^{0}\right), \eta_{0} \geqslant 1\right] .
\end{aligned}
$$


We need now only to bound appropriately the two terms in the last line. Note that the second term is handled exactly as in case 1 .

For the first term, some additional notation is required. Define the set $E=\left\{\eta: \mid T_{n, l}-\right.$ $\left.\rho^{n, l} \mid / \sqrt{c^{n, l}} \leqslant M\right\}$ for fixed $M$ and note that $Z_{\alpha}\left\{E^{c}\right\} \leqslant M^{-2}$. Let also $\xi=\tau_{n, l}(\eta)$ so that in particular $\xi_{x_{n, l}}=T_{n, l}$. Denote $Z_{\alpha(\cdot), n, l}$ as the induced measure of $\xi$ with respect to $Z_{\alpha(\cdot)}$ and $E_{\alpha(\cdot), n, l}$ as its expectation. Denote also $\mu_{\alpha(\cdot), n, l}$ as the induced measure corresponding to $\xi_{x_{n, l}}$. Note that $Z_{\alpha(\cdot), n, l}$ is the product measure $\prod_{i \notin A_{n, l}} \mu_{\alpha(i)} \times \prod_{\substack{i \in A_{n, l} \\ i \neq x_{n, l}}} \mu_{0} \times \mu_{\alpha(\cdot), n, l}$. The following change of measure from $\xi$ to $\xi^{x_{n, l}, 0}$ will be useful:

$$
\begin{aligned}
\frac{d Z_{\alpha(\cdot), n, l}\left\{\xi^{x_{n, l}, 0}\right\}}{d Z_{\alpha(\cdot), n, l}\{\xi\}} & =\frac{\mu_{\alpha(0)}\left(\xi_{0}+1\right)}{\mu_{\alpha(0)}\left(\xi_{0}\right)} \frac{\mu_{\alpha(\cdot), n, l}\left(\xi_{x_{n, l}}-1\right)}{\mu_{\alpha(\cdot), n, l}\left(\xi_{x_{n, l}}\right)} \\
& =\frac{\alpha(0)}{g\left(\xi_{0}+1\right)} \frac{Z_{\alpha(\cdot)}\left\{\zeta: \sum_{i \in A_{n, l}} \zeta_{i}=\xi_{x_{n, l}}-1\right\}}{Z_{\alpha(\cdot)}\left\{\zeta: \sum_{i \in A_{n, l}} \zeta_{i}=\xi_{x_{n, l}}\right\}} .
\end{aligned}
$$

Let also $\rho_{i}=E_{\alpha(\cdot)}\left[\eta_{i}\right]$ and $\sigma_{i}^{2}=E_{\alpha(\cdot)}\left[\left(\eta_{i}-\rho_{i}\right)^{2}\right]$. Define $\rho^{n, l}=\sum_{i \in A_{n, l}} \rho_{i}$ and $c^{n, l}=$ $\sum_{i \in A_{n, l}} \sigma_{i}^{2}$. Let also $\gamma^{n, l}=\sum_{i \in A_{n, l}} E_{\alpha(\cdot)}\left[\left|\eta_{i}-\rho_{i}\right|^{3}\right]$ and $p_{i j}=Z_{\alpha(\cdot)}\left\{\eta_{i}=j\right\}$.

Now split the expectation $E_{\alpha(\cdot)}\left[R^{2}\left(\left(\tau_{n, l}(\eta)\right)^{0, x_{n, l}}\right), \eta_{0} \geqslant 1\right]$ into

$$
E_{\alpha(\cdot)}\left[R^{2}\left(\left(\tau_{n, l}(\eta)\right)^{0, x_{n, l}}\right), \eta_{0} \geqslant 1, E\right]+E_{\alpha(\cdot)}\left[R^{2}\left(\left(\tau_{n, l}(\eta)\right)^{0, x_{n, l}}\right), \eta_{0} \geqslant 1, E^{c}\right]=J_{1}+J_{2}
$$

respectively. As $\|R\|_{\infty}<C_{f}$, we have $J_{2}<C(f, \alpha, g) M^{-2}$.

Rewrite now the term $J_{1}$ as

$$
\begin{aligned}
J_{1} & =E_{\alpha(\cdot), n, l}\left[R^{2}\left(\xi^{0, x_{n, l}}\right), \xi_{0} \geqslant 1, E\right] \\
& =E_{\alpha(\cdot), n, l}\left[\frac{\alpha(0)}{g\left(\xi_{0}+1\right)} \frac{Z_{\alpha(\cdot)}\left\{\zeta: \sum_{i \in A_{n, l}} \zeta_{i}=\xi_{x_{n, l}}-1\right\}}{Z_{\alpha(\cdot)}\left\{\zeta: \sum_{i \in A_{n, l}} \zeta_{i}=\xi_{x_{n, l}}\right\}} R^{2}(\xi), E^{\prime}\right],
\end{aligned}
$$

where we change variables $\xi$ to $\xi^{x_{n, l}, 0}$, and $E^{\prime}=\left\{\xi:\left|\xi_{x_{n, l}}-1-\rho^{n, l}\right| / \sqrt{c^{n, l}} \leqslant M, \xi_{x_{n, l}} \geqslant\right.$ $1\}$.

To estimate $J_{1}$ further, we invoke Proposition 2.2. To apply the proposition, we show that the occupation numbers in $A_{n, l}$ satisfy the hypotheses: (1) Note that $p_{i 0}=$ $Z(\alpha(i))^{-1} \rightarrow 1$ as $\alpha(i)$ vanishes for $|i| \uparrow \infty$ and $p_{i j} \leqslant(\alpha(i) / I)^{j} Z(\alpha(i))^{-1} \leqslant p_{i 0}$ as soon as $\alpha(i) \leqslant I$. As $\alpha(i) \rightarrow 0$ we may choose $n$ so that, for the variables indexed in $A_{n, l}$, the first condition on $p_{i j}$ is satisfied. (2) Explicit calculation gives that

$$
\rho_{i}=\frac{1}{Z(\alpha(i))} \frac{\alpha(i)}{g(1)}+\frac{\alpha(i)^{2}}{Z(\alpha(i))} \sum_{k \geqslant 2} \frac{k \alpha(i)^{k-2}}{(g(k) \cdots g(1))} .
$$

Note, as $U=0$, that $\alpha_{\max }=\max \{\alpha(i), i \in S\}<\infty$ exists. It is not difficult now to conclude that there are constants $C^{\prime}(\alpha, g)>C(\alpha, g)>0$ such that for large $n$ and $l>n$ that

$$
C^{\prime} \sum_{i \in A_{n, l}} \alpha(i) \leqslant \rho^{n, l}, c^{n, l}, \gamma^{n, l} \leqslant C \sum_{i \in A_{n, l}} \alpha(i)
$$


This together with the fact that $\sum_{i \in A_{n, l}} \alpha(i)$ diverges as $l$ increases gives the second condition. (3) To see that the g.c.d. is 1 we show that 1 is in the set. Calculate $p_{i 0} p_{i 1}=Z(\alpha(i))^{-2} \cdot \alpha(i) / g(1)$. For $n$ large enough, we have that $\sum_{i \in A(n, l)} p_{i 0} p_{i 1}$ is equivalent to $c^{n, l}$. The fact that $c^{n, l}$ diverges with $l$ gives the last condition.

We now apply Proposition 2.2 to estimate the fraction $Z_{\alpha(\cdot)}\left\{\zeta: \sum_{i \in A_{n, l}} \zeta_{i}=\xi_{x_{n, l}}-\right.$ $1\} / Z_{\alpha(\cdot)}\left\{\zeta: \sum_{i \in A_{n, l}} \zeta_{i}=\xi_{x_{n, l}}\right\}$ on the set $E^{\prime}$. The error term in the proposition is absolutely bounded by $C(\alpha, g) / \sqrt{c^{n, l}}$ for some constant $1<C(\alpha, g)<\infty$, say. For $l$ large, this error may be bounded by $C(\alpha, g, M)$. We see now that the fraction above on the set $E^{\prime}$ is bounded above by another $C(\alpha, g, M)$.

Putting these estimates together gives the bound

$$
E_{\alpha(\cdot)}\left[R^{2}\left(\tau_{n, l}(\eta)\right), \eta_{0} \geqslant 1\right]<C(\alpha, g, f) M^{-2}+C(\alpha, g, M) E_{\alpha(\cdot), n, l}\left[R^{2}(\xi)\right] .
$$

Now $E_{\alpha(\cdot)}\left[R^{2}(\eta)\right]=E_{\alpha(\cdot), n, l}\left[R^{2}(\xi)\right]$ so that $C(\alpha, g, f) M^{-2}+C(\alpha, g, M) \varepsilon^{2}$ is a further bound which is as small as we want. This finishes the proof.

Proof of Proposition 2.1. -

"(b) $\Rightarrow$ (c)" Let $Q$ be an invariant measure whose path measure is ergodic. Write $Q=\varepsilon Q_{1}+(1-\varepsilon) Q_{2}$ for $0<\varepsilon<1$ and $Q_{1}$ and $Q_{2}$ invariant measures. It follows that both $Q_{1}$ and $Q_{2}$ are absolutely continuous with respect to $Q$. Let now $f$ be a bounded function. Then, as $t \rightarrow \infty, \frac{1}{t} \int_{0}^{t}\left(T_{s} f\right) d s$ converges in probabilty to $E_{Q}[f]$ and $\hat{f}$ with respect to $Q$ and $Q_{1}$ respectively. By absolute continuity, $\hat{f}=E_{Q}[f] Q_{1}$-a.s., and so $E_{Q_{1}}[f]=E_{Q_{1}}[\hat{f}]=E_{Q}[f]$. This gives that $Q_{1}(B)=Q(B)$ for $B \in \mathcal{B}$ and therefore $Q_{1}=Q$.

"(a) $\Rightarrow$ (b)" Let $Q$ be an invariant measure and suppose that $P^{Q}$ is not ergodic. Then there exists an $f \in L^{2}(Q)$ such that $\hat{f}$ is not constant $Q$-a.s. Let $c$ be such that $Q(A)=\varepsilon, 0<\varepsilon<1$ where $A=\{\hat{f}>c\}$. Now, as $T_{t} \hat{f}=\hat{f} Q$-a.s. and $T_{t}$ is a positive contraction taking 1 into 1 , we have that $T_{t} I(A)=I(A) Q$-a.s.: First, as $T_{t}$ is a positive operator, $|\hat{f}|=\left|T_{t} \hat{f}\right| \leqslant T_{t}|\hat{f}|$, so that, as $T_{t}$ is an $L^{2}$ contraction, we have that $Q$-a.s. $T_{t}|\hat{f}|=|\hat{f}|$. Therefore, $\max \{0, \hat{f}\}=(\hat{f}+|\hat{f}|) / 2$ is harmonic. Further, if $f, g \in L^{2}$ are harmonic, then $\max \{f, g\}=\max \{f-g, 0\}+g$ is harmonic. Correspondingly, $\min \{f, g\}=-\max \{-f,-g\}$ is harmonic. Of course, 1 is harmonic. All of this gives that $\min \{n \max (0, \hat{f}-c), 1\}$ for $n \geqslant 1$ is a sequence of bounded harmonic functions. The limit, as $n \rightarrow \infty$, is $I(A)$ which is therefore harmonic by dominated convergence. This proves the implication.

"(c) $\Rightarrow$ (a)" Let $A$ be such that $T_{t} I(A)=I(A) Q$-a.s. and $Q(A)=\varepsilon$ for $0<\varepsilon<1$. As the process begun on $A$ stays in $A$ with $Q$-probability 1 , we have that $Q_{1}(B)=$ $\varepsilon^{-1} Q(B \cap A)$ and $Q_{2}(B)=(1-\varepsilon)^{-1} Q\left(B \cap A^{c}\right)$ are distinct invariant measures such that $Q=\varepsilon Q_{1}+(1-\varepsilon) Q_{2}$. Therefore $Q$ is not extremal.

This finishes the proof.

\section{Applications}

We remark in this section on some consequences of Theorem 1.4. Consider the following two problems. 


\section{Invariance principle}

Let $f(\eta)$ be a local function on the state space $\Sigma$. With respect to initial configurations distributed by $B_{\rho}$ or $Z_{\alpha}$ for which functions $f$ does

$$
\frac{1}{\sqrt{\lambda}} \int_{0}^{\lambda t} f(\eta(s)) d s \rightarrow B\left(\sigma^{2}(f) t\right)
$$

for $\sigma^{2}(f)<\infty$ as $\lambda \rightarrow \infty$ ? Given extremality of $B_{\rho}$ or $Z_{\alpha}$ and under some conditions on $p$ and the rate $g$, this problem was resolved in [17]. With $Z_{\alpha}$ now seen to be extremal in more cases than before, we note the corollary to Theorem 1.4:

COROLlARY 3.1. - Corollary 1.1 of [17] is valid in all dimensions, not only as before in dimensions $d=1,2$ when extremality of $Z_{\alpha}$ was not clear in higher dimensions.

Please see [17] for more details.

\section{Tagged particle motion}

Consider a zero-range system on $Z^{d}$ with initial distribution governed by $Z_{\alpha}\left\{\cdot \mid \eta_{0} \geqslant\right.$ $1\}$ for $\alpha$ constant; in other words, a distingued, or "tagged" particle is placed initially at the origin. Let $x(t)$ be the position of the tagged particle at time $t$ (note $x(0)=0$ ) and let $\rho(\alpha)=E_{\alpha}\left[\eta_{1}\right]$, the mean particle density at a site. Also assume the jump probabilities are irreducible, translation invariant, $p(i, j)=p(j-i)$. Saada proves in [16] for zerorange dynamics with rate $g(k) \equiv I(k \geqslant 1)$, that (a) if $\sum|i| p(i)<\infty$, then $Z_{\alpha}$-a.s.,

$$
x(t) / t \rightarrow(1-\rho(\alpha)) \sum i p(i)
$$

and (b) if $p$ is mean-zero and $\sum|i|^{2} p(i)<\infty$, then with respect to $Z_{\alpha}$,

$$
x(t) / \sqrt{t} \rightarrow N(0,(1-\rho(\alpha)) \Gamma),
$$

where the $d$-dimensional covariance $\Gamma=\left(\sum_{z \in Z^{d}} z_{i} z_{j} p(z)\right)$. The proof relies on the extremality given in parts (2a) and (2b) of Theorem 1.2.

The same proof for general $g$, noting part (1a) of Theorem 1.4, applies to yield the corollary:

COROLLARY 3.2. - Consider the tagged particle zero-range system above with $g$ satisfying $(Z R)$.

(a) If $\sum|i| p(i)<\infty$, then $Z_{\alpha}$-a.s.,

$$
x(t) / t \rightarrow(\alpha / \rho(\alpha)) \sum i p(i) .
$$

(b) If $p$ is mean-zero and $\sum|i| p(i)<\infty$, then with respect to $Z_{\alpha}$,

$$
x(t) / \sqrt{t} \rightarrow N(0,(\alpha / \rho(\alpha)) \Gamma),
$$

where $\Gamma$ is the d-dimensional covariance, $\Gamma=\left(\sum_{z \in Z^{d}} z_{i} z_{j} p(z)\right)$. 


\section{Acknowledgement}

I would like to thank Ellen Saada for many useful, pleasant conversations. I would like also to thank E. Andjel, N. Jain, S. Olla, J. Sethuraman and S. Varadhan for useful discussions. I would also like to thank the referee for careful and helpful comments. In addition, thanks to Niati Sanzu for help with French translation.

\section{REFERENCES}

[1] Albeverio S., Kondratiev Y.G., Rockner M., Ergodicity of $L^{2}$-semigroups and extremality of Gibbs states, J. Funct. Anal. 144 (1997) 394-423.

[2] Aldous D., Pitman J., On the zero-one law for exchangeable events, Ann. Probab. 7 (1979) 704-723.

[3] Andjel E.D., Invariant measures for the zero range process, Ann. Probab. 10 (1982) 525547.

[4] Andjel E., Cocozza-Thivent C., Roussignol M., Quelqes complements sur le processus des misanthropes et le processus "zero-range", Ann. Inst. Henri Poincare 21 (4) (1985) 363 382.

[5] Cocozza-Thivent C., Processus des misanthropes, Z. Wahr. Verw. Gebiete 70 (1985) 509523.

[6] Ethier S., Kurtz T., Markov Processes: Characterization and Convergence, Wiley, New York, 1986.

[7] Georgii H.O., Canonical Gibbs Measures, Lecture Notes in Mathematics, Vol. 760, Springer-Verlag, Berlin, 1979.

[8] Guo M.Z., Papanicolaou G., Self-diffusion of interacting brownian particles, in: Prob. Methods in Math. Phys., Taniguchi Symp. Katata/Kyoto, Academic Press, 1985, pp. 113151.

[9] Liggett T.M., Random invariant measures for Markov chains, and independent particle systems, Z. Wahr. Verw. Gebiete 45 (1978) 297-313.

[10] Liggett T.M., Interacting Particle Systems, Springer-Verlag, New York, 1985.

[11] Mineka J., A criterion for tail events for sums of independent random variables, Z. Wahr. Verw. Gebiete 25 (1973) 163-170.

[12] Petrov V.V., Sums of Independent Random Variables, Springer-Verlag, New York, 1975.

[13] Rosenblatt M., Transition probability operators, in: Proc. Fifth Berkeley Symp. Math. Statist. Prob., Vol. 2, 1967, pp. 473-483.

[14] Rosenblatt M., Markov Processes. Structure and Asymptotic Behavior, Springer-Verlag, New York, 1971.

[15] Saada E., A limit theorem for the position of a tagged particle in a simple exclusion process, Ann. Probab. 15 (1) (1987) 375-381.

[16] Saada E., Processus de zero-range avec particule marquee, Ann. Inst. Henri Poincare 26 (1) (1990) 5-17.

[17] Sethuraman S., Xu L., A central limit theorem for reversible exclusion and zero-range particle systems, Ann. Probab. 24 (4) (1996) 1842-1870.

[18] Spohn H., Large Scale Dynamics of Interacting Particles, Springer-Verlag, Berlin, 1991.

[19] Waymire E., Zero range interaction at Bose-Einstein speeds under a positive recurrent single particle law, Ann. Probab. 8 (1980) 441-450. 